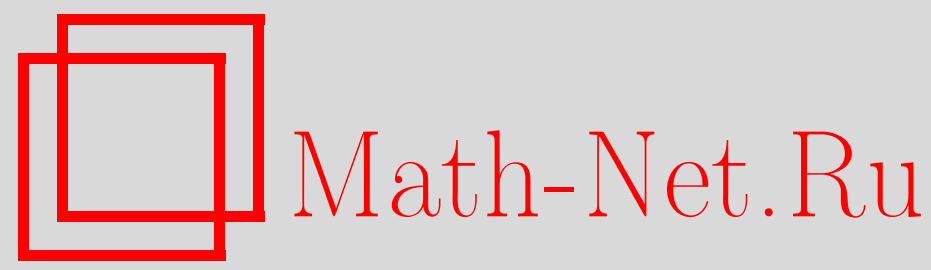

В. П. Скляров, Об одном полиномиальном неравенстве Г. Фройда, Матем. заметки, 1996, том 60, выпуск 5, 788-792

DOI: https://doi.org/10.4213/mzm1895

Использование Общероссийского математического портала MathNet.Ru подразумевает, что вы прочитали и согласны с пользовательским соглашением

http://www . mathnet.ru/rus/agreement

Параметры загрузки:

IP : 54.205 .225 .156

26 апреля 2023 г., 15:42:01






\section{ОБ ОДНОМ ПОЛИНОМИАЛЬНОМ НЕРАВЕНСТВЕ Г. ФРОЙДА}

\section{В.П. Скляров}

В 1971 году Г. Фройдом в [1] было доказано следующее утверждение: ecлu $\left|p_{n}(x)\right| \leqslant e^{x^{2} / 2} n p u|x| \leqslant(1+\varepsilon) \sqrt{2 n}$, mо для всех $x\left|p_{n}(x)\right| \leqslant C_{1}(\varepsilon) e^{x^{2} / 2}$.

Здесь $p_{n}(x)$ - любой алгебраический многочлен степени не выше $n, C_{1}(\varepsilon)$ - положительное число, которое может зависеть разве лишь от $\varepsilon, \mathbf{a} \varepsilon>0$ может быть любым. Очевидно, что это утверждение эквивалентно неравенству

$$
\max _{-\infty \leqslant x \leqslant \infty}\left|p_{n}(x)\right| e^{-x^{2} / 2} \leqslant C_{1}(\varepsilon) \max _{|x| \leqslant R_{n}}\left|p_{n}(x)\right| e^{-x^{2} / 2},
$$

где $R_{n}=(1+\varepsilon) \sqrt{2 n}$. В начале 80 -х годов нашего столетия Х.Н. Маскар и Е.Б. Сафф получили усиленные аналоги результата Фройда для различных весов и пространств. В частности, для этого случая ими было доказано, что неравенство (1) можно заменить равенством

$$
\max _{-\infty \leqslant x \leqslant \infty}\left|p_{n}(x)\right| e^{-x^{2} / 2}=\max _{|x| \leqslant R_{n}}\left|p_{n}(x)\right| e^{-x^{2} / 2},
$$

в котором в качестве значения $R_{n}$ может быть использована константа Рахманова-Маскара-Саффа: $a_{n}=\sqrt{2 n}$. Все связанные с этим детали можно найти в работах Маскара и Сафффа [2], [3]. Ниже будет показано, что величина $\sqrt{2 n}$ не является наименьшей в соотношении $(2)$, а неуменьшаемое значение $R_{n}$ совпадает с наибольшей точкой чебьшевского альтернанса для многочлена, наименее уклоняющегося от нуля на всей вещественной оси с весом $e^{-x^{2} / 2}$.

Начнем с рассмотрения конкретного случая $n=1$. Очевидно, что тогда равенство (2) достаточно рассмотреть для многочленов вида $p_{1}(x)=x-x_{1}$. Легко видеть, что

$$
\max _{-\infty \leqslant x \leqslant \infty}\left|p_{1}(x)\right| e^{-x^{2} / 2}=\max \left\{\left|p_{1}\left(s_{1}\right)\right| e^{-s_{1}^{2} / 2},\left|p_{1}\left(s_{2}\right)\right| e^{-s_{2}^{2} / 2}\right\},
$$

где $s_{1}, s_{2}$ - корни уравнения

$$
x p_{1}(x) e^{-x^{2} / 2}=e^{-x^{2} / 2} .
$$

Функция $\frac{1}{s} e^{-s^{2} / 2}$ убывает при $s>0$, поэтому, предположив, что $\left|s_{1}\right| \leqslant\left|s_{2}\right|$, из (3) находим

$$
\max _{-\infty \leqslant x \leqslant \infty}\left|p_{1}(x)\right| e^{-x^{2} / 2}=\left|p_{1}\left(s_{1}\right)\right| e^{-s_{1}^{2} / 2} .
$$

Очевидно, что (4) эквивалентно квадратному уравнению $x^{2}-x_{1} x^{2}-1=0$, следовательно, $s_{1} s_{2}=-1$ и $\left|s_{1}\right| \leqslant 1$ при любом $x_{1}$. Таким образом, для любого многочлена степени не выше первой имеет место равенство (2) с величиной $R_{1}=1$, то, что полученное значение не может быть уменьшено, подтверждает полином $p_{1}(x)=x$. Следовательно, при $n=1$ константа Рахманова-Маскара-Саффа не является оптимальной. Следующее утверждение показьвает, что равенство (2) останется справедливым при любом натуральном $n$ для значений $R_{n}<a_{n}$. 
ТЕОРема 1. Для любого алгебраического многочлена $p_{n}(x)$ nри $n \geqslant 1$ имеет место равенство

$$
\max _{-\infty \leqslant x \leqslant \infty}\left|p_{n}(x)\right| e^{-x^{2} / 2}=\max _{|x| \leqslant \sqrt{2 n-1}}\left|p_{n}(x)\right| e^{-x^{2} / 2} .
$$

ДоКАЗАТЕЛЬСтво. Пусть $x_{1}<x_{2}<\cdots<x_{n}$ - нули многочлена Эрмита $H_{n}(x)$, определяемого равенством

$$
H_{n}(x)=(-1)^{n}\left(2^{n} n ! \sqrt{\pi}\right)^{-1 / 2} e^{x^{2}} \frac{d^{n}}{d x^{n}}\left(e^{-x^{2}}\right) .
$$

Положим по определению $x_{n+1}=-x_{0}=\sqrt{2 n+1}$ и обозначим $L_{n}[F](x)-$ интерполяционный многочлен Лагранжа, построенный для функции $F(x)$ по $n+2$ узлам $x_{0}, x_{1}, \ldots, x_{n+1}$. Тогда для любого алгебраического многочлена $p_{n+1}(x)$ будем иметь

$$
\begin{aligned}
\left|p_{n+1}(x)\right| e^{-x^{2} / 2} & =\left|L_{n}\left[p_{n+1}(x)\right](x)\right| e^{-x^{2} / 2} \\
& \leqslant \Lambda_{n}(x) \max _{|x| \leqslant x_{n+1}}\left\{\left|p_{n+1}(x)\right| e^{-x^{2} / 2}\right\} .
\end{aligned}
$$

Здесь

$$
\begin{aligned}
\Lambda_{n}(x)= & \sum_{\nu=1}^{n}\left|\frac{\left(x^{2}-x_{n+1}^{2}\right) \varphi_{n}(x)}{\left(x_{\nu}^{2}-x_{n+1}^{2}\right) \varphi_{n}^{\prime}\left(x_{\nu}\right)\left(x-x_{\nu}\right)}\right| \\
& +\frac{\left|x-x_{n+1}\right|+\left|x+x_{n+1}\right|}{2 x_{n+1}} \cdot\left|\frac{\varphi_{n}(x)}{\varphi_{n}\left(x_{n+1}\right)}\right|
\end{aligned}
$$

a

$$
\varphi_{n}(x)=H_{n}(x) e^{-x^{2} / 2} .
$$

Наша ближайшая цель - проверка справедливости равенства

$$
\max _{|x| \geqslant x_{n+1}} \Lambda_{n}(x)=1 .
$$

Сначала заметим, что $\varphi_{n}^{\prime}(x)$ есть результат умножения многочлена степени $n+1$ на экспоненциальный вес, поэтому

$$
\varphi_{n}^{\prime}(x)=L_{n}\left[\varphi_{n}^{\prime}(x) e^{x^{2} / 2}\right] e^{-x^{2} / 2} .
$$

Развернутая запись этого равенства дает

$$
\varphi_{n}^{\prime}(x)=\sum_{\nu=1}^{n} \frac{\left(x^{2}-x_{n+1}^{2}\right) \varphi_{n}(x)}{\left(x_{\nu}^{2}-x_{n+1}^{2}\right)\left(x-x_{\nu}\right)}+\frac{x \varphi_{n}(x)}{x_{n+1} \varphi_{n}\left(x_{n+1}\right)} \cdot \varphi_{n}^{\prime}\left(x_{n+1}\right) .
$$

Отсюда находим, что

$$
\sum_{\nu=1}^{n} \frac{\left(x^{2}-x_{n+1}^{2}\right) \varphi_{n}(x)}{\left(x_{n+1}^{2}-x_{\nu}^{2}\right)\left(x-x_{\nu}\right)}=-\varphi_{n}^{\prime}(x)+\frac{x \varphi_{n}(x)}{x_{n+1} \varphi_{n}\left(x_{n+1}\right)} \cdot \varphi_{n}^{\prime}\left(x_{n+1}\right) .
$$


В силу симметричного расположения узлов интерполирования и убывания величин $\left|\varphi_{n}^{\prime}\left(x_{\nu}\right)\right|$ с ростом номера $\nu$ при $x_{\nu} \geqslant 0$ [4] заключаем для $x \geqslant x_{n+1}$, что

$$
\Lambda_{n}(x) \leqslant\left|\varphi_{n}^{\prime}\left(x_{n}\right)\right|^{-1} \sum_{\nu=1}^{n} \frac{\left(x^{2}-x_{n+1}^{2}\right) \varphi_{n}(x)}{\left(x_{n+1}^{2}-x_{\nu}^{2}\right)\left(x-x_{\nu}\right)}+\frac{x \varphi_{n}(x)}{x_{n+1} \varphi_{n}\left(x_{n+1}\right)} \cdot \varphi_{n}^{\prime}\left(x_{n+1}\right) .
$$

Отсюда, учитывая (7), легко заметить, что

$$
\Lambda_{n}(x) \leqslant \frac{x \varphi_{n}(x)}{x_{n+1} \varphi_{n}\left(x_{n+1}\right)}-\frac{\varphi_{n}^{\prime}(x)}{\left|\varphi_{n}^{\prime}\left(x_{n}\right)\right|}+\frac{x \varphi_{n}(x)}{x_{n+1} \varphi_{n}\left(x_{n+1}\right)} \cdot \frac{\varphi_{n}^{\prime}\left(x_{n+1}\right)}{\left|\varphi_{n}^{\prime}\left(x_{n}\right)\right|} .
$$

Дифференциальное уравнение для функций Эрмита $\varphi_{n}^{\prime \prime}(x)+\left(2 n+1-x^{2}\right) \varphi_{n}(x)=0$ позволяет утверждать, что $\varphi_{n}^{\prime}(x)$ возрастает при $x \geqslant x_{n+1}$, поэтому $\varphi_{n}^{\prime}\left(x_{n+1}\right)<$ $\varphi_{n}^{\prime}(x)$ и из $(8)$ находим

$$
\Lambda_{n}(x) \leqslant \frac{x \varphi_{n}(x)}{x_{n+1} \varphi_{n}\left(x_{n+1}\right)}-\frac{\varphi_{n}^{\prime}(x)}{\left|\varphi_{n}^{\prime}\left(x_{n}\right)\right|}\left[1-\frac{x \varphi_{n}(x)}{x_{n+1} \varphi_{n}\left(x_{n+1}\right)}\right] .
$$

Рекуррентные формулы и формулы дифференцирования для многочленов Эрмита легко приводят к равенству $x \varphi_{n}(x)=-\varphi_{n}^{\prime}(x)+\varphi_{n-1}(x) \sqrt{2 n}$, из которого получаем $\left(x \varphi_{n}(x)\right)^{\prime}=\left(2 n+1-x^{2}\right) \varphi_{n}(x)+\varphi_{n-1}^{\prime}(x) \sqrt{2 n}<0$, конечно же при $x \geqslant x_{n+1}$. Из этого заключаем, что при рассматриваемых значениях $x$ имеет место неравенство

$$
\frac{x \varphi_{n}(x)}{x_{n+1} \varphi_{n}\left(x_{n+1}\right)} \leqslant 1 .
$$

Поскольку $\left|\varphi_{n}^{\prime}\left(x_{n}\right)\right| \geqslant\left|\varphi_{n}^{\prime}\left(x_{n+1}\right)\right|$, то с помошью (9) имеем

$$
\begin{aligned}
\Lambda_{n}(x) & \leqslant \frac{x \varphi_{n}(x)}{x_{n+1} \varphi_{n}\left(x_{n+1}\right)}+\frac{\left|\varphi_{n}^{\prime}\left(x_{n+1}\right)\right|}{\left|\varphi_{n}^{\prime}\left(x_{n}\right)\right|}\left[1-\frac{x \varphi_{n}(x)}{x_{n+1} \varphi_{n}\left(x_{n+1}\right)}\right] \\
& \leqslant \frac{x \varphi_{n}(x)}{x_{n+1} \varphi_{n}\left(x_{n+1}\right)}+1-\frac{x \varphi_{n}(x)}{x_{n+1} \varphi_{n}\left(x_{n+1}\right)}=1 .
\end{aligned}
$$

Из (5) следует четность функции $\Lambda_{n}(x)$, поэтому $\max _{|x| \geqslant x_{n+1}} \Lambda_{n}(x) \leqslant 1$. Однако, $\Lambda_{n}\left(x_{n+1}\right)=1$, следовательно, $\max _{|x| \geqslant x_{n+1}} \Lambda_{n}(x)=1$. Очевидно, что

$$
\begin{aligned}
\max _{-\infty \leqslant x \leqslant \infty}\left|p_{n+1}(x)\right| e^{-x^{2} / 2} \\
=\max \left\{\max _{|x| \leqslant x_{n+1}}\left|p_{n+1}(x)\right| e^{-x^{2} / 2} ; \max _{|x| \geqslant x_{n+1}}\left|p_{n+1}(x)\right| e^{-x^{2} / 2}\right\},
\end{aligned}
$$

но, учитывая (5), приходим к неравенству

$$
\max _{-\infty \leqslant x \leqslant \infty}\left|p_{n+1}(x)\right| e^{-x^{2} / 2}=\max _{|x| \leqslant \sqrt{2 n+1}}\left|p_{n+1}(x)\right| e^{-x^{2} / 2} .
$$

Заменив здесь $n$ на $n-1$, получим утверждение теоремы.

Можно показать, что $\sqrt{2 n-1}$ также не является оптимальным значением параметра $R_{n}$ в соотношении (2). Чтобы указать это оптимальное значение, предположим

$$
\inf _{p_{n-1}}\left\|x^{n}-p_{n-1}(x)\right\|=\left\|G_{n}(x)\right\| \quad \text { и } \quad\|f(x)\|=\max _{-\infty \leqslant x \leqslant \infty}|f(x)| e^{-x^{2} / 2} .
$$

Тогда в силу существования чебышевского альтернанса [5] уравнение $\left(G_{n}(x) \times\right.$ $\left.e^{-x^{2} / 2}\right)^{\prime}=0$ имеет $n+1$ корень. Занумеруем эти корни в порядке возрастания $s_{0}<s_{1}<\cdots<s_{n}$. 
Теорема 2. Значение параметра $R_{n}=s_{n}$ является наименьшим среди тех, при которых равенство (2) остается справедливым для любого алгебраического многочлена степени $n$.

ДоКАЗАТЕЛЬСтво. Проверка того, что равенство (2) остается справедливым при $R_{n}=s_{n}$ для любого алгебраического многочлена степени $n$ фактически повторяет доказательство предыдущей теоремы, поэтому, опуская детали, заметим, что если в роли узлов интерполирования выбираются точки $s_{\nu}$, то для функции Лебега $\Lambda_{n}(x)$ при $x \geqslant s_{n}$ будет иметь место равенство

$$
\Lambda_{n}(x)=\frac{\left|G_{n}(x)\right| e^{-x^{2} / 2}}{\left\|G_{n}\right\|} \leqslant 1 .
$$

Чтобы проверить вторую часть утверждения теоремы 2 о минимальности $s_{n}$, рассмотрим отображение, действующее из $\mathbb{R}^{n}$ в $\mathbb{R}^{n+1}$ следующим образом. Вектору $\bar{x}\left(x_{1}, x_{2}, \ldots, x_{n}\right)$ поставим в соответствие вектор $\bar{m}\left(m_{0}, m_{1}, \ldots, m_{n}\right)$, определяя его координаты с помощью многочлена $P_{n}(x)=\left(x-x_{1}\right)\left(x-x_{2}\right) \cdots\left(x-x_{n}\right)$ равенствами

$$
\begin{aligned}
m_{0} & =\max _{x \leqslant x_{1}}\left|P_{n}(x)\right| e^{-x^{2} / 2} \\
m_{i} & =\max _{x_{i} \leqslant x \leqslant x_{i+1}}\left|P_{n}(x)\right| e^{-x^{2} / 2}, \quad i=1,2, \ldots, n-1, \\
m_{n} & =\max _{x_{n} \leqslant x}\left|P_{n}(x)\right| e^{-x^{2} / 2} .
\end{aligned}
$$

Это отображение определено в каждой точке открытого симплекса $x_{1}<x_{2}<$ $\cdots<x_{n}$ и дифференцируемо. В самом деле, если корни $t_{\nu}$ уравнения

$$
\left(P_{n}(x) e^{-x^{2} / 2}\right)^{\prime}=0
$$

занумерованы в порядке возрастания, то

$$
m_{i}=\left|P_{n}\left(t_{i}\right)\right| e^{-t_{i}^{2} / 2}=\left(x_{n}-t_{i}\right) e^{-t_{i}^{2} / 2} \prod_{k=1}^{n-1}\left|t_{i}-x_{k}\right|, \quad i=0,1, \ldots, n-1
$$

Аналогично,

$$
m_{n}=\left|P_{n}\left(t_{n}\right)\right| e^{-t_{n}^{2} / 2}=\left(t_{n}-x_{n}\right) e^{-t_{n}^{2} / 2} \prod_{k=1}^{n-1}\left(t_{n}-x_{k}\right)
$$

Отсюда, учитывая, что $t_{i}=t_{i}\left(x_{1}, x_{2}, \ldots, x_{n}\right)$ удовлетворяют уравнению (10), находим при $i=0,1, \ldots, n-1$

$$
\frac{\partial m_{i}}{\partial x_{n}}=e^{-t_{i}^{2} / 2} \prod_{k=1}^{n-1}\left|t_{i}-x_{k}\right|>0
$$

а при $i=n$

$$
\frac{\partial m_{n}}{\partial x_{n}}=e^{-t_{n}^{2} / 2} \prod_{k=1}^{n-1}\left(t_{n}-x_{k}\right)<0
$$


Следовательно, если мы у многочлена $G_{n}(x)$ произведем достаточно малый сдвиг влево только одного корня $x_{n}$, то для нового многочлена $\widehat{G}_{n}(x)$ будем иметь $\left\|\widehat{G}_{n}\right\|=m_{n}$, а при $i=0,1, \ldots, n-1$ будем иметь $m_{i}<m_{n}$. Поскольку точки $t_{i}$ непрерьвно зависят от $x_{n}$, то, таким образом, для любого $\varepsilon>0$ мы можем построить многочлен $\widehat{G}_{n}(x)$ так, что его норма будет достигаться только в одной точке $t_{n}$, и эта точка будет лежать на отрезке $\left[s_{n}-\varepsilon, s_{n}\right]$. Этим доказана вторая часть теоремы 2 .

Нетрудно заметить, что утверждение этой теоремы останется в силе для произвольного промежутка $[a, b]$ и дифференцируемого веса $\rho(x)$ такого, что $\rho(a)=$ $\rho(b)=0$, а при $x \in(a, b) \rho(x)>0$.

И еше одно замечание. Возможность использования величины $s_{n}$ в равенстве (2) была доказана ранее в [2, теорема 2.1] другим способом, однако, вопрос о неуменьшаемости этого значения там не рассматривался.

Саратовский государственный университет

Поступило

E-mail: sklyarov@scnit.saratov.su

07.02 .96

\section{СПИСОК ЦИТИРОВАННОЙ ЛИТЕРАТУРЫ}

1. Freud G. // Acta Math. Acad. Sci. Hung. 1971. V. 22. № 1-2. P. 109-116. 2. Mhaskar H. N., Saff E. B. // Trans. Amer. Math. Soc. 1984. V. 285. P. 203-234. 3. Mhaskar H. N., Saff E. B. // Constr. Approx. 1985. V. 1. P. 71-91. 4. Скляров В. П. // Изв. вузов. Матем. 1993. № 11. С. 57-61. 5. Дзядык В. К. Введение в теорию равномерного приближения функций полиномами. М.: Наука, 1977. 